\title{
InSight: the interplay of science and humanities
}

InSight: Visualizing Health Humanities

Edited by Bonnie Sadler Takach, Pamela

Brett-MacLean, Aidan Rowe and Geo

Takach

University of Alberta; 2012

$\mathrm{I}$ $\mathrm{n}$ the foreword to InSight: Visualizing Health Humanities, Allan Bleakley, the renowned humanities scholar, refers to psychoanalyst, Donald Winnicott's idea that "social play is essential to developing empathy" and relates this idea to the role of the humanities in medicine. He believes that by creating a "potential space," where adults can "play," the humanities are able to provide "a place for imagination to be exercised." This in turn, can help a person develop empathy and tolerance for uncertainty, essentially leading to democratization of medicine.

Bleakley draws on Martha Nussbaum, the American philosopher's argument that "humanities diagnose social ills such as groundless and unproductive authoritarian behavior, and suggest cures such as tolerance of difference through open debate." In this light, Bleakley envisions a potential role for humanities in shifting the culture of medicine toward greater acceptance of the limits of scientific knowledge, openness to ambiguities in diagnosis and treatment and essentially less paternalism in medical decision-making. This, he refers to as "the democratic project," within the field of medicine.

The InSight art exhibit, which was the basis of this publication, was an attempt to create the kind of open dialogue between sciences and the humanities through the spirit of play as suggested by Bleakley. Held at the University of Alberta as part of the Arts \& Humanities in Health and Medicine program, the exhibit comprised 32 artworks by students and staff. It was indeed full of playful pieces that invited the audience to join in the creation of meaning through stretching imagination.

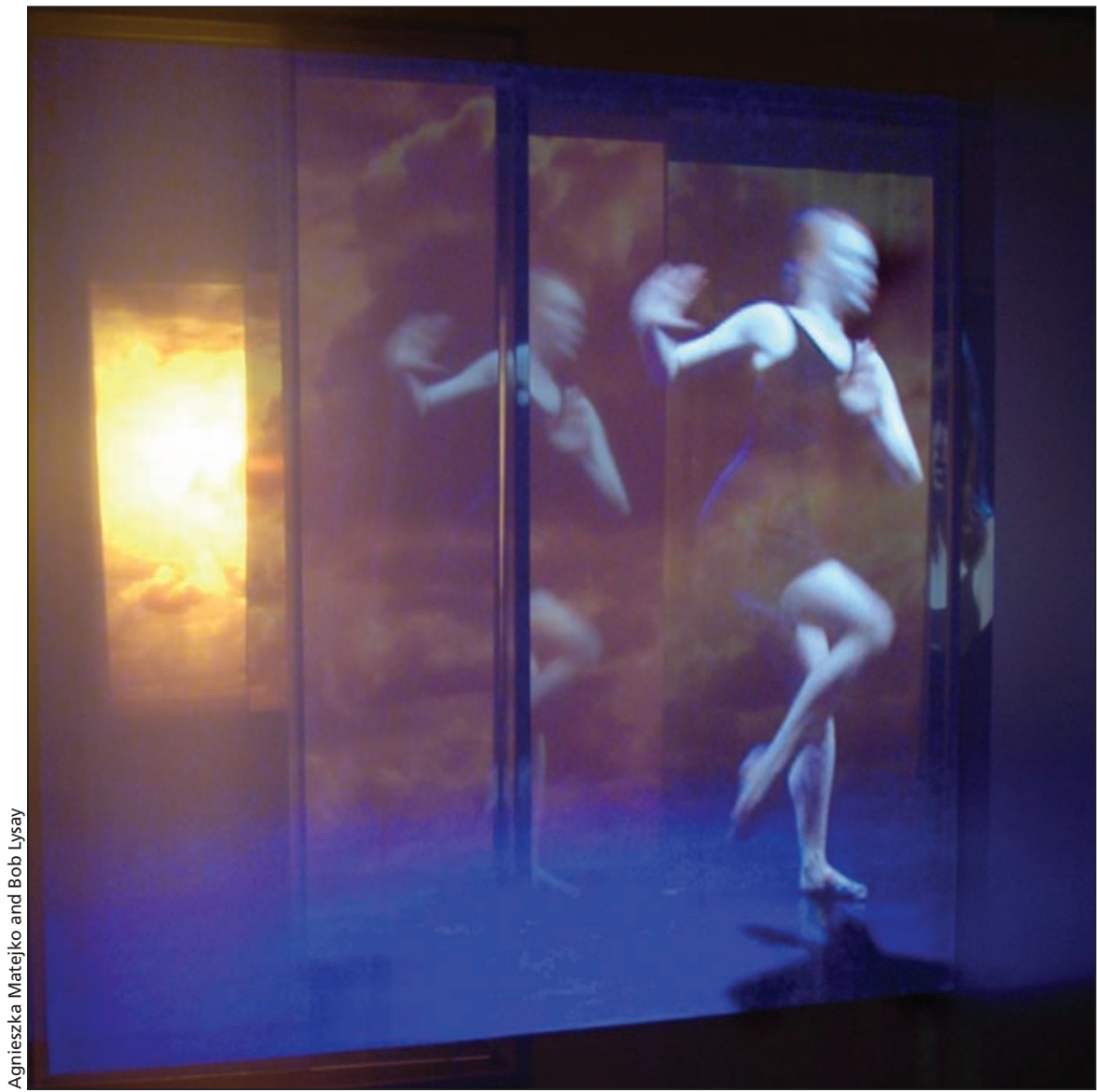

The Space Between You and Me is a visual art installation depicting the insight of youth with Tourette syndrome.

The publication strives to capture this playful atmosphere with colourful, captivating images, and the detailed descriptions of the audiovisual installations aid in bringing the interactive pieces to life. Moreover, the artistic use of small images at the bottom of pages that recount the previous artwork and foreshadow the next, mimic the act of walking through an art gallery. There is, furthermore, a disjointed aspect to the arrangement of the content that invites the reader to create personal links and find meaning between and through the pieces. For a reader who has not had a chance to see the exhibit, the book can serve as a virtual tour.

Serpens Oleum: The Phantasmagoric Amphigorium of Dr. Wybury, is one example of the artworks depicted in the book. It consists of a curious, antique wooden cabinet decorated with Victorian medicine bottles, snake oils, pieces of vertebrae and teeth and other medical equipment. A computer screen has been installed in the middle of the top shelf, which presents random "pseudo-medical proclamations" related to modern medical sciences when a lever is pulled. By placing "a modern source of information" within the context of "Victorian medical culture," the artists Marlena Wyman and Chris Westbury argue that the piece raises "questions about the relation between contemporary medical facts and the discarded and discredited, almost comically ignorant, medical 'facts' of earlier times." Thus, the artists cast doubt on the validity of what may be known 


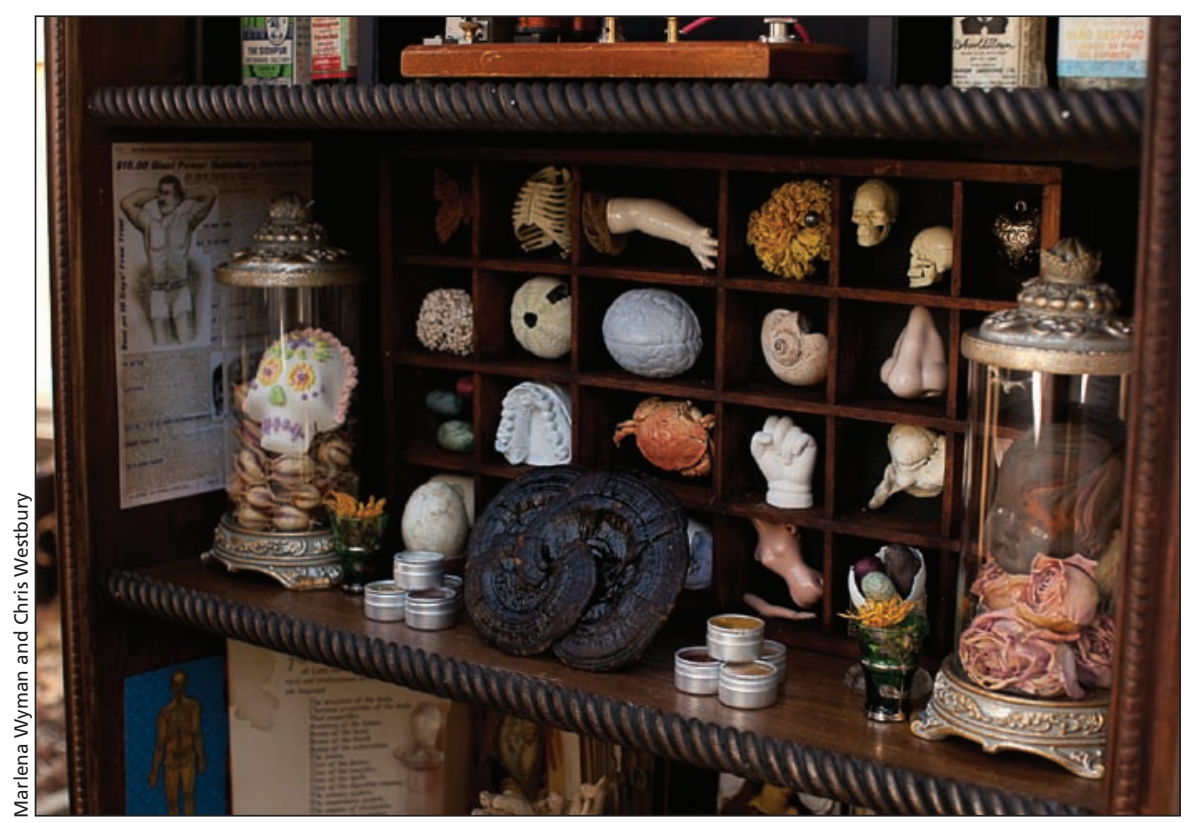

Serpens Oleum: The Phantasmagoric Amphigorium of Dr. Wybury is an interactive art installation offering a wry commentary on the "snake oils" of the 21st century.

today as a "scientific fact," questioning the certainty of scientific knowledge.

This type of dialogue between medicine and humanities, which places some of the most basic assumptions in the medical field under scrutiny, is characteristic of many of the artworks depicted in this book. In another piece, The Space Between You \& Me, a widely different perspective on what is described as a neuropsychiatric diagnosis is offered. In this installation, seven youths with Tourette syndrome share their insights about living with this disorder, while a young dancer performs an original choreography interpreting the move- ments typically associated with Tourette syndrome. According to the artists, this artwork explores "the beauty, poignancy and inspiring insight of youth with disorders" and reconsiders the medical definition of a "dis-order." Critical outlook of these art pieces, which speaks to what Bleakely describes as the "democratizing force" of the humanities, is a feature that makes the publication compelling and thought provoking.

Given the disjointed quality of the content, Bleakley's editorial serves an integrating function, which places the book and the exhibit into perspective. Whether creating a potential space for "play" in arts and humanities can eventually help in fostering what he advocates in his foreword (i.e., empathy, tolerance for ambiguity and democratization of medicine) among health professionals, is a question that cannot be easily answered. Nonetheless, the dialogue that projects such as the InSight exhibition open between medical sciences and the arts and humanities is an important starting point for us as it fosters curiosity about what these disciplines have to teach us about health care and healing.

\section{Mehr-Afarin Hosseini MD \\ Resident in psychiatry \\ University of Toronto \\ Toronto, Ont.}

For additional images see Appendix 1, available at www.cmaj.ca/lookup/suppl /doi:10.1503/cmaj.121868/-/DC1

\section{CMAJ 2013. DOI:10.1503/cmaj.121868}

Follow-up exhibit: InSight2: Engaging the Health Humanities took place from May 14-June 8, 2013. This international exhibition and accompanying book and exhibit catalogue extends inquiry into the evolving field of health humanities, and examine linkages, experiences, visualisations and productive imaginings at the nexus of health humanities, design and community engagement. For more details visit www.insight 2.healthhumanities.ca 